\title{
Wideband, Multiband, Tunable, and Smart Antenna Systems for Mobile and UWB Wireless Applications
}

\author{
Renato Cicchetti, ${ }^{1}$ Antonio Faraone, ${ }^{2}$ Diego Caratelli, ${ }^{3}$ and Massimiliano Simeoni ${ }^{4}$ \\ ${ }^{1}$ Department of Information, Electronic and Telecommunication Engineering, University of Rome "La Sapienza", Rome, Italy \\ ${ }^{2}$ Chief Technology Office, Motorola Solutions Inc., Fort Lauderdale, FL 33322, USA \\ ${ }^{3}$ Microwave Technology and Systems for Radar, Delft University of Technology, The Netherlands \\ ${ }^{4}$ European Space Agency, ESTEC-Keplerlaan, ZH Noordwijk, The Netherlands
}

Correspondence should be addressed to Renato Cicchetti; cicchetti@die.uniromal.it

Received 6 August 2013; Accepted 6 August 2013

Copyright (C) 2013 Renato Cicchetti et al. This is an open access article distributed under the Creative Commons Attribution License, which permits unrestricted use, distribution, and reproduction in any medium, provided the original work is properly cited.

\section{Introduction}

With the advent of high data rate $3 \mathrm{G}$ and $4 \mathrm{G}$ wireless communication systems and the app-based use paradigm, wireless connectivity through multiple air interfaces has become a common requirement in the RF architecture of new generation mobile communication devices. The modern wireless handset easily incorporates three or more antennas to enable cellular, $\mathrm{Wi}-\mathrm{Fi}$, and GPS connectivity, frequently over multiple bands. Multiple antenna systems are frequently designed to implement diversity or spatial multiplexing schemes, as in the case of WCDMA and LTE, to increase the resiliency and capacity of wireless links, and even to operate multiple voice/data links simultaneously. Concurrently, ultrawideband (UWB) systems used in short range communications, remote sensing, and through-the-wall radar imaging have introduced a new paradigm in antenna design where the mitigation of pulse distortion is of the essence, thus requiring a shift in antenna design approach and the introduction of novel radiating systems.

This special issue is intended to reflect current $R \& D$ trends and novel approaches in the analysis and synthesis of antenna systems for the new generation of mobile communication devices, such as smartphones, tablets, and laptop computers as well as for UWB communication systems and radars. A particular emphasis has been paid to the analysis and design of broadband, multiband, and reconfigurable antennas for wireless and UWB applications, as well as to the identification of integration techniques with the host platform. Important efforts have been devoted to the characterization of the radio channel for MIMO systems.

The special issue is composed of 18 contributions that can be divided into the following 8 clusters.

\section{Contributions to Reconfigurable and Multiband Antenna Technology}

In "Recent developments in reconfigurable and multiband antenna technology" by N. Haider et al., the authors present a comparative analysis of various reconfigurable and multiband antenna concepts. In particular, three basic approaches, tunable/switchable antenna integration with radiofrequency switching devices, wideband or multiband antenna integration with tunable filters, and array architectures with the same aperture utilized for different operational modes, have been analyzed in detail showing inherent benefits and challenges.

In "A reconfigurable triple-Notch-Band antenna integrated with defected microstrip structure band-stop filter for ultrawideband cognitive radio applications" by $\mathrm{Y}$. Li et al., the authors describe a reconfigurable UWB monopole antenna for cognitive radio applications. Three narrow band-notched frequencies are obtained using a defected microstrip structure (DMS), a stop band filter (BSF) embedded in the microstrip feed line, and an inverted $\pi$-shaped slot etched in the rectangular radiant patch, respectively. Reconfigurable 
characteristics of the proposed cognitive radio antenna are achieved by means of four ideal switches integrated on the DMS-BSF and the inverted $\pi$-shaped slot.

In "Planar ultrawideband antenna with photonically controlled notched bands" by D. Draskovic et al., the authors describe a planar microstrip-fed UWB printed circular monopole antenna with optically controlled notched bands. The proposed antenna is composed of a circular UWB patch, with an etched T-shaped slot controlled by an integrated optical switch. The slot modifies the frequency response of the antenna suppressing the $3.5-5 \mathrm{GHz}$ band when the switch is in an open state. The optical switch is controlled by a lowpower laser diode rendering the antenna remotely controlled by means of an optical fiber.

In "Compact, frequency reconfigurable, printed monopole antenna" by R. Gonçalves et al., the authors propose, a compact reconfigurable printed monopole antenna, useful to operate in the UMTS and WLAN bands. To this purpose a chip inductor and PIN diode are employed to modify the electrical length of the monopole making the antenna able to operate in the mentioned frequency bands.

\section{Contributions to Broad- and Multibanding Techniques}

In "Analysis and design of a novel compact multiband printed monopole antenna" by J. Wang and X. He, the authors present a compact T-shaped multiband printed monopole antenna integrating some U-shaped band-notch structures. The proposed antenna operates at $2.25-2.7 \mathrm{GHz}, 3.25-3.6 \mathrm{GHz}, 4.95-$ $6.2 \mathrm{GHz}$, and $7-8 \mathrm{GHz}$, covering the operation bands of Bluetooth, WiMAX, and WLAN and the downlink of the Xband satellite communication systems.

In "Dual-band integrated antennas for DVB-T receivers" by A. D'alessandro et al., the authors present an overview on compact Planar Inverted-F Antennas (PIFAs) that are suitable for monitor-equipped devices. High efficiency PIFAs with a percentage bandwidth greater than $59 \%(470-862 \mathrm{MHz}$ DVB-T band) are shown. Finally, to show the extreme flexibility of the previous two configurations, a novel dualband L-shape PIFA has been proposed. The L-shape PIFA prototype is obtained by properly cutting and folding a single metal sheet, thus resulting in a relatively low-cost and mechanically robust antenna configuration.

In "Planar printed shorted monopole antenna with coupled feed for LTE/WWAN mobile handset applications" by D. Kang and Y. Sung, the authors present a shorted monopole antenna with coupled feed for LTE/WWAN mobile handset applications. The basic resonance of the shorted monopole combines with the resonance caused by the interaction between the coupling strip and the feeding pad to cover the LTE700, GSM850, and GSM900 bands. Both the feeding pad and the coupling strip operate with the shorting strip as a loop antenna. The resonance of the loop antenna and the harmonics of the shorted monopole combine to cover the GSM1800, GSM1900, UMTS, and LTE2300 bands. A stable and omnidirectional radiation pattern with reasonable gain has been observed over the operating bandwidth.
In "Parasitic-element-loaded UWB antenna with bandstop function for mobile handset wireless USB" by Y. Lim et al., the authors present an antenna loaded by parasitic elements for the wireless USB of mobile handsets useful for UWB service in which a band-stop function of 5.725$5.825 \mathrm{GHz}$ WLAN band is required. To this end, two kinds of parasitic elements are incorporated into a rectangular radiator to obtain enhanced impedance bandwidth and bandstop function. In this way, bandwidths of $3.15-4.75 \mathrm{GHz}$ and of 7.2-10.2 GHz are achieved, while the frequency band 5.725$5.825 \mathrm{GHz}$ is suppressed.

\section{Contributions to Antennas for UWB Applications}

In "A compact UWB antenna with a quarter-wavelength Strip in a Rectangular slot for $5.5 \mathrm{GHz}$ band notch" by P. Moeikham et al., the authors present a monopole UWB antenna integrating a notch filter to reduce the electromagnetic interferences (EMIs) with WLAN/WiMAX communication systems operating in the frequency band located around $5.5 \mathrm{GHz}$. Consisting in a rectangular slot including a quarter-wavelength strip integrated on the lower inner edge of the UWB radiating patch, the filter proposed by the authors is capable of reducing the energy emission in the frequency range between 5.1 and $5.75 \mathrm{GHz}$ resulting in lower EMIs with sensible electronic equipment working in this frequency band.

In "A modified vivaldi antenna for improved angulardependent fidelity property" by Z. Zeng et al., the authors present a modified Vivaldi antenna optimized to radiate impulsive signals. To this end, a spatial filter consisting of two suitable dielectric slabs parallel to the antenna substrate is introduced. As a result, the fidelity factor indicating the quality of the radiated field is significantly improved. In fact, the experimental measurements show that the ranges with the fidelity factor better than the value of 0.9 are improved by $95 \%$ in $\mathrm{H}$-plane and by $14 \%$ in E-plane, respectively, with respect to a conventional Vivaldi antenna.

In "High gain compact strip and slot UWB sinuous antennas" by E. Agastra et al., the authors analyze three groundbacked compact strip and slot sinuous antennas. The proposed configuration allows for a single lobe, polarizationversatile, high efficiency, and ultrawideband antenna not needing a cumbersome lossy back cavity typical of conventional single-lobe sinuous antennas.

\section{Contributions to Special Materials, and Fabrication Techniques}

In "Innovative radiating systems for train localization in interference conditions" by C. Vegni et al., the authors, firstly, propose an innovative radiating systems based on the metamaterial technology for global navigation satellite system (GNSS) applications in radio frequency interference conditions. Secondly, fixed radiation pattern antenna (FRPA), and controlled radiation pattern antenna (CRPA) phased array configurations of miniaturized patch antennas are studied. 
Finally, the design of the phased array is applied to a GNSS user receiver in a realistic railway environment.

\section{Contributions to Wireless Systems for Remote Control of Vital Parameters}

In "A twin spiral planar antenna for UWB medical radars" by G. A. Zito et al., the authors propose a planar-spiral antenna useful to be employed in an UWB radar system for heart activity monitoring. The proposed antenna presents a reflection coefficient lower than $-8 \mathrm{~dB}$ over the $3-12 \mathrm{GHz}$ band, while the coupling between the two spiral dipoles is about $-20 \mathrm{~dB}$. Numerical simulations indicate that the radiation impedance variation, caused by the thorax vibrations associated with heart activity, is the most likely explanation of the UWB radar operation.

In "Wireless sensing for the respiratory activity of human beings: measurements and wide-band numerical analysis" by L. Scalise et al., the authors propose an RF sensing system for the remote control of the respiratory activity. The proposed sensing system is based on the measurement of the phase variation of the reflection coefficient caused by the respiratory activity. The phase signal compared with the thorax displacement measured by a reference instrument shows a high correlation with different subject postures (sitting, standing, and lying), and a reduction of the signal amplitude with the distance $-0.11 \mathrm{~dB} / \mathrm{cm}$ is reported. The proposed system makes it possible to operate at distances up to $2.5 \mathrm{~m}$.

In "Safety aspects of people exposed to ultra wideband radar fields" by M. Cavagnaro et al., the authors analyze the safety aspects of people exposed to a field emitted by UWB radar for breath activity monitoring, operating both in the spatial environment and on ground. The basic restrictions and reference levels reported in the ICNIRP safety guideline are considered, and the compliance of electromagnetic fields radiated by a UWB radar with these limits has been evaluated. The authors show that if the field emitted by the UWB radar is compliant with spatial and/or ground emission masks, then both reference levels and basic restrictions are largely satisfied.

\section{Contributions to Numerical and Analytical Techniques for Antenna Modeling and Design}

In "Structure-based evolutionary programming design of broadband wire antennas" by G. A. Casula et al., the authors present a designed technique for wideband wire antennas. The technique, based on the structure-based evolutionary programming, is used to design a broadband antenna, operating in the $3-16 \mathrm{GHz}$ frequency band, with an end-fire radiation pattern, high gain, good impedance matching, and robustness with respect to realization tolerances.

\section{Contributions to Passive Devices per UWB Applications}

In "Optimized ultrawideband and uniplanar minkowski fractal branch line coupler" by M. Jahanbakht and M. T. Aghmyoni, the authors present a directional coupler, based on a nonEuclidean Minkowski fractal geometry able to operate with excellent isolation and low insertion losses over the UWB frequency range. The proposed device presents good integration features that make it suitable to be employed as a power divider in electronic circuitry or in the array of broadband or UWB antennas.

\section{Contributions to MIMO Antenna Systems and Channel Modeling}

In "Sparse channel estimation for MIMO-OFDM two-way relay network with compressed sensing" by A. Zhang et al., the authors propose a sparse channel estimation scheme at end users under the relay channel to enable us to exploit sparsity. First, they formulate the sparse channel estimation problem as a compressed sensing problem by using the sparse decomposition theory. Second, the CIR is reconstructed by CoSaMP and OMP algorithms. The numerical results confirm the superiority of the proposed methods over the traditional linear channel estimation methods.

\section{Acknowledgments}

The editors would like to express their gratitude to the authors and the anonymous reviewers for their contributions to this special issue.

Renato Cicchetti

Antonio Faraone

Diego Caratelli

Massimiliano Simeoni 

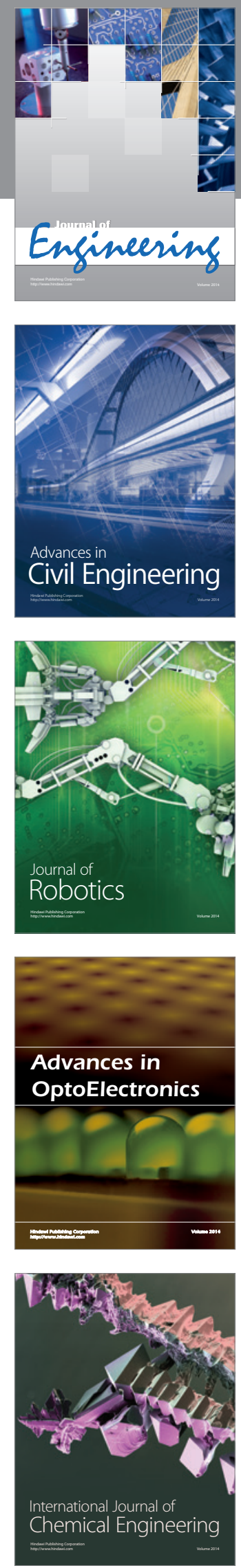

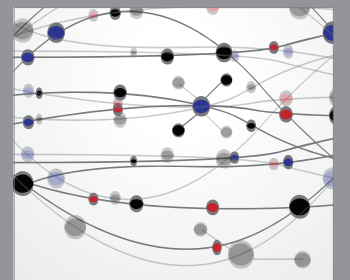

The Scientific World Journal
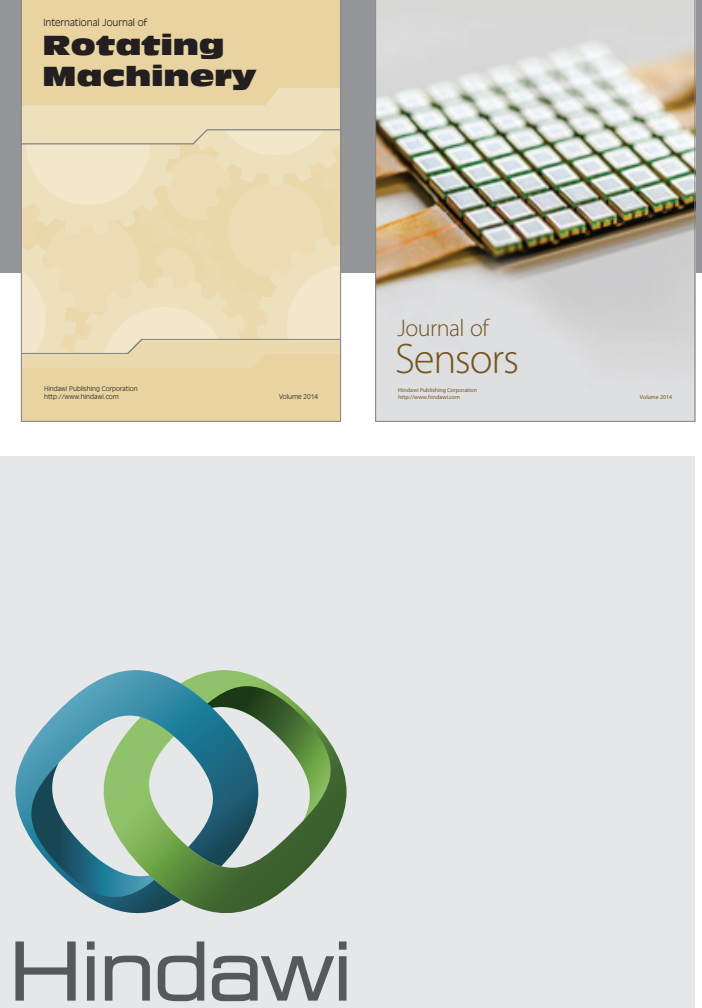

Submit your manuscripts at http://www.hindawi.com
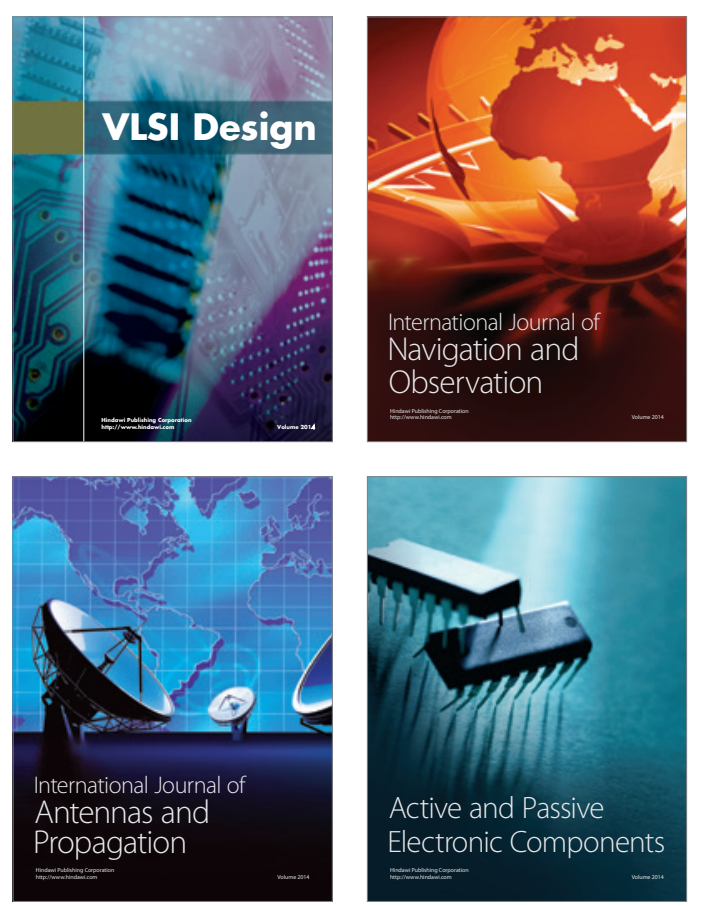
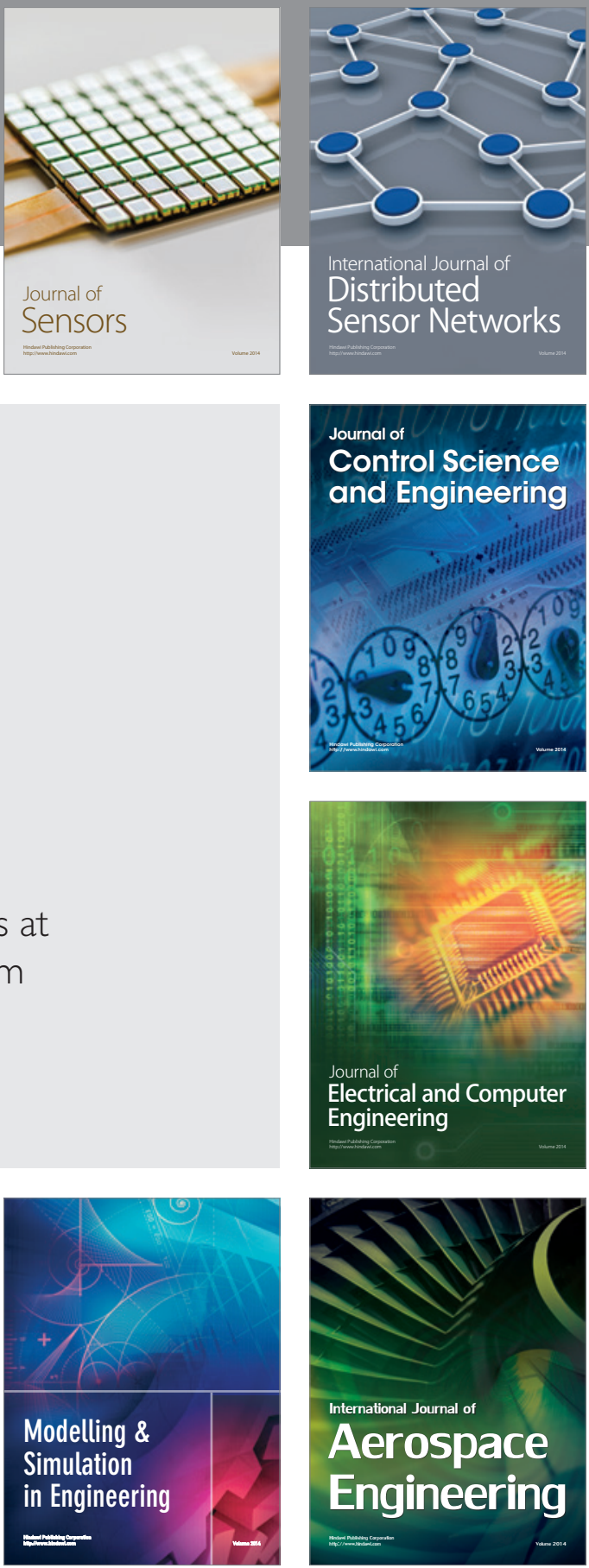

Journal of

Control Science

and Engineering
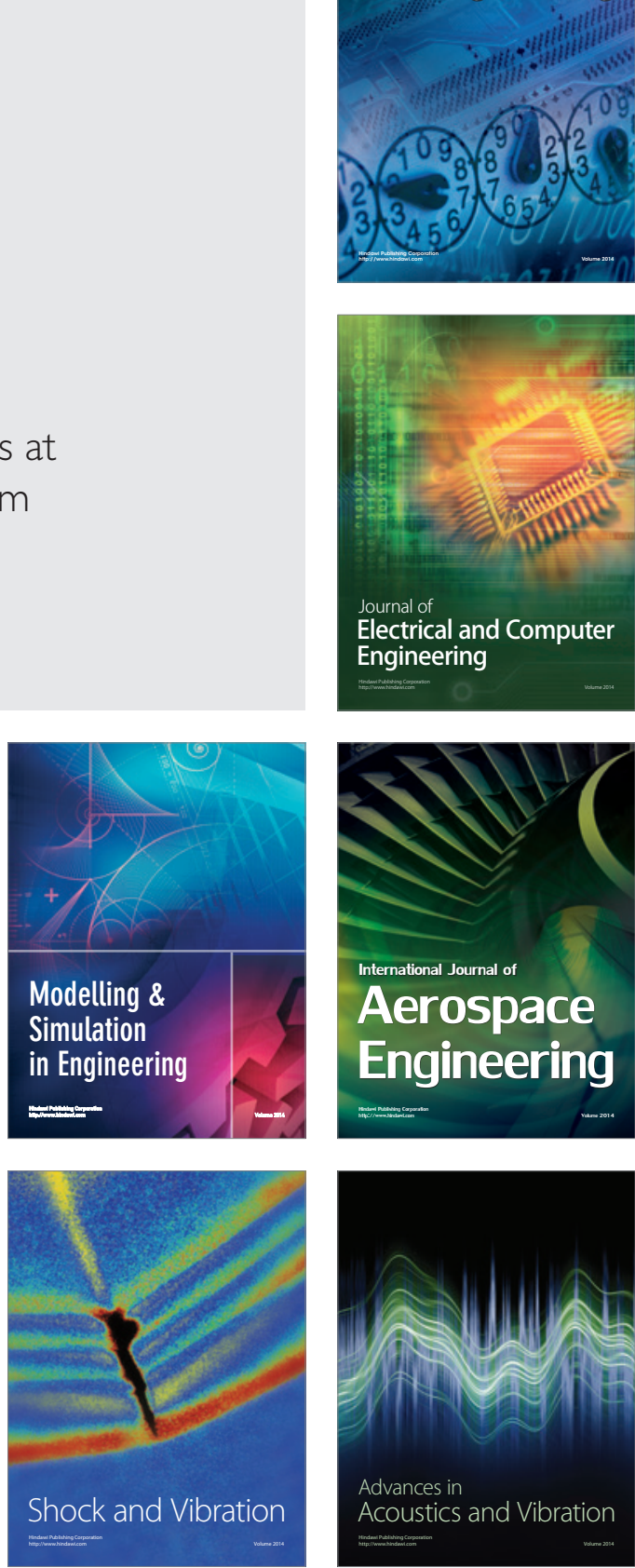